\title{
基于双向渐进结构优化法的柔性机构设计 ${ }^{*}$
}

\author{
何健 何猛夏凉史铁林 \\ (华中科技大学数字制造装备与技术国家重点实验室 武汉 430074)
}

\begin{abstract}
摘要: 基于双向渐进结构优化法提出一种面向柔性机构的拓扑优化设计策略。由于采用 $0 / 1$ 离散拓扑设计变量和启发式变量 更新机制, 双向渐进结构优化法一般适用于结构刚度相关的凸优化设计问题。柔性机构以最大化驱动端的位移为设计目标, 属于典型的非凸优化问题, 难以直接应用该方法开展相应的设计。针对于此, 通过定义一种由驱动位移和刚度特性(柔顺度) 加权平均的优化目标函数, 实现基于双向渐进结构优化法的柔性机构设计。该优化目标函数具有双重功效: (1) 通过逐步（设 计迭代步)衰减刚度特性的贡献, 实现优化问题由刚度设计向驱动设计的动态演化, 可应用双向渐进结构优化法开展机构的拓 扑构型设计; (2) 通过调节加权系数, 实现对设计机构的驱动性能和刚度特性的灵活匹配调控, 可有效抑制铰链的形成、防 止应力集中引起的失效。典型算例的设计结果显示, 提出的发展的演化式设计策略可实现稳健且高效的柔性机构设计。
\end{abstract}

关键词: 柔性机构; 拓扑优化; 双向渐进结构优化法; 动态演化; 驱动设计

中图分类号: $\mathrm{O} 343$

\section{Design of Compliant Actuation Mechanisms by Evolutionary Structural Optimization Method}

\author{
HE Jian HE Meng XIA Liang SHI Tielin
}

(State Key Laboratory of Digital Manufacturing Equipment and Technology, Huazhong University of Science and Technology, Wuhan 430074)

\begin{abstract}
This research proposes a topology optimization design strategy for compliant actuation mechanisms using the evolutionary structural optimization (ESO) method. Because of the discrete nature of design variables (0/1) and heuristic update mechanism, ESO-type methods have been mainly applied for convex optimization problems such as structural stiffness maximization design. Compliant mechanisms design with maximized actuated displacements as the design objective is a typical non-convex problem and ESO methods cannot be applied directly. In this regard, a weighted objective function is proposed by combining actuated displacement and structural stiffness (in terms of compliance). The merits of the newly defined objective function are twofold. First, by gradually (design iterations) damping the stiffness contribution, the design objective dynamically evolves from stiffness to actuation, and thus ESO methods can be applied. Second, a flexible control on actuation and stiffness performances can be achieved by adjusting the weights, which assists in avoiding the appearance of fragile hinges and prevents failure due to stress concentration. By means of a series of benchmark design tests, the proposed evolutionary design strategy has been shown robust and high-efficient in designing compliant actuation mechanisms.
\end{abstract}

Key words : compliant mechanism; topology optimization; ESO; dynamic evolution; actuation design

\section{0 前言}

柔性机构通常是指通过弹性变形将输入的力或 位移传递到驱动端的一类整体式结构。由于其整体

* 国家自然科学基金(11972166)、湖北省自然科学基金杰出青年基金 (2020CFA080)和中央高校基本科研业务费专项资金(2019kfyXKJC044) 资助项目。20201014 收到初稿, 20210204 收到修改稿
式的结构特性, 柔性机构具有无需组装、无摩擦、 节省空间等优点，在医药和微机电等领域有广泛的 应用 ${ }^{[1-2]}$ 。由于柔性机构的诸多性能优势, 近几十年 中, 已经对柔性机构的设计进行了大量研究并取得 了长足的发展。

柔性机构的主要设计方法有: 基于运动学的方 法 $^{[3]}$, 构件方法 ${ }^{[4]}$ 和拓扑优化方法 ${ }^{[5-6]}$ 。其中, 拓扑 优化通过在设计域中优化材料布局形成高效的结构 
形式, 具有极高的设计效率和自由度, 被广泛应用 于柔性机构的设计 ${ }^{[7-8]}$ 。目前主流的拓扑优化方法 有: 变密度法 ${ }^{[9]}$ 、水平集法 ${ }^{[10]}$ 、渐进结构优化法 ${ }^{[11-12]}$ 、 和移动可变形组件法 ${ }^{[13]}$ 等。柔性机构拓扑优化设计 的目标主要有两类: 第一类为最大化某些机械测量 值, 如: 输出(驱动)端位移 ${ }^{[14]}$ 、输出与输入端的位 移比值(几何增益 ${ }^{[15]}$ )、载荷比值(机械增益 ${ }^{[16]}$ )、以及 功率比值 ${ }^{[17]}$ 等; 另一类则综合机构刚度和驱动性 能, 通过权重因子协调各目标间的比重, 如: 2014 年, 朱本亮等 ${ }^{[18]}$ 提出一种同时优化驱动位移和两种 平均柔度的设计目标; 2017 年, CHU 等 ${ }^{[19]}$ 提出综 合输出位移和机构刚度来设计柔性机构; 2020 年, LIU 等 ${ }^{[20]}$ 在设计中考虑了实际输出位移。

早期利用拓扑优化设计的柔性机构, 其结构构 型中一般难以避免会出现铰链现象。铰链在结构中 起类刚体的作用, 可近似看作旋转关节、最大化地 传递运动。然而, 铰链区域往往存在应力集中, 容 易发展断裂失效; 同时不易于制造, 在设计中应尽 量避免 ${ }^{[21]}$ 。近年来, 除却通过设计以最大化柔性机 构的驱动性能, 越来越多的研究开始关注如何同步 提高机构的刚度性能、避免铰链的形成, 以提高驱 动的稳健性。较为直接的避免铰链的方法可在后处 理阶段对铰链区域进行二次设计 ${ }^{[22]}$ 。一般而言, 二 次设计与初始设计机构的性能存在较大的偏差。此 外, 还可以通过改进过滤方式 ${ }^{[23]}$ 、施加最小尺寸约 束 $^{[24]}$ 、施加应力约束 ${ }^{[25]}$ 等方法来避免铰链的形成。 此外, 研究发现引入刚度性能指标的多目标优化模 型, 能够较好地避免铰链的形成。结构的刚度性能 可由应变能定量表征(应变能越小、刚度越大)。通 过将设计目标定义为耦合驱动变形和结构应变能的 加权形式 ${ }^{[26-27]}$, 或在驱动设计中考虑应变能约 束 ${ }^{[28-29]}$, 可实现机构驱动设计的同时, 有效提高机 构的刚度、避免铰链的形成。

在已发展诸多主流拓扑优化设计方法中, 于 1992 年提出的渐进结构优化法 ${ }^{[11]}$ 是一类基于仿生 演化机制的启发式算法, 通过逐步去除低效材料 演化形成较优的结构构型。根据优化问题的定义, 材料的服役效率可定义为位移、变形、应力或能 量等形式的结构响应。黄晓东等 ${ }^{[30]}$ 基于该方法后 期提出了设计稳健性更强的双向渐进结构优化 法, 即在拓扑构型迭代的过程中材料可双向增减。 相较于其他优化方法, 渐进结构优化法采用离散 设计变量 $(0 / 1)$ 和启发式变量更新机制, 概念简单明 晰、设计效率高、设计构型的材料边界清晰, 已 广泛应用于静动力学、多物理场、多尺度等领域
的结构优化设计研究 ${ }^{[31]}$, 并且已在工程领域得到 初步的推广应用 ${ }^{[32-34]}$ 。

值得一提的是, 尽管双向渐进结构优化法已在 多个设计领域得到成功应用, 目前鲜有应用该方法 开展柔性机构设计的文献报道 ${ }^{[35]}$ 。2007 年, ANSOLA 等 ${ }^{[36]}$ 曾提出从空材料设计域逐步增补材 料的渐进式设计策略, 实现柔性机构的设计; 然而, 该设计策略缺乏可解释结构拓扑构型变化的基于物 理模型的驱动机理, 且难以额外考虑刚度性能的约 束。之后, 黄晓东等 ${ }^{[37]}$ 于 2014 年改进了双向渐进 结构优化法以实现柔性机构的设计; 其中, 设定单 元密度设计变量以某一设定的步长( $2 \%$ ) 在 0 和 1 之 间阶跃变化, 同时考虑结构的刚度作为设计约束。 通过调试驱动变形与刚度约束的权重系数, 该方法 可实现无铰链柔性机构的设计。显然, 允许密度设 计变量在 0 和 1 之间阶跃变化的处理策略, 是与传 统渐进结构优化法的变量离散特性(0/1)相违背的; 因此, 不属于双向渐进结构优化法在柔性机构设计 问题上的典型应用。

本文通过定义一种由驱动位移和刚度特性(柔 顺度)加权平均的优化目标函数, 通过逐步(设计迭 代步)衰减刚度特性的贡献, 实现优化问题由刚度设 计向驱动设计的动态演化, 实现基于双向渐进结构 优化法的柔性机构设计。其次，借鉴朱本亮等 ${ }^{[18]}$ 的 思想, 通过调节驱动位移和刚度特性的加权系数抑 制铰链的形成。全文编排如下：第 1 节定义适用于 双向渐进结构优化法的柔性机构设计目标函数形 式; 第 2 节介绍拓扑优化列式和灵敏度计算格式; 第 3 节通过两个典型算例验证所发展设计策略的有 效性; 第 4 节总结全文。

\section{1 动态演化的加权目标函数}

图 1 为单输入-输出的柔性机构设计问题的示 意图。柔性机构的工作模式为: 设计域内, 在输入 端施加驱动载荷, 通过机构的弹性变形传递运动, 在输出端产生驱动变形。线弹性小变形模型假设下, 柔性机构的设计可简化为输入端施加单位载荷, 通 过机构构型 $(\Omega)$ 的设计, 最大化输出端驱动方向的 位移。将机构进行有限元离散, 输入端单位载荷作 用下的整体机构的位移响应可由下式计算得到

$$
\boldsymbol{K} \boldsymbol{U}_{\text {in }}=\boldsymbol{F}_{\text {in }}
$$

式中, $\boldsymbol{K}$ 为机构的整体刚度矩阵, $\boldsymbol{F}_{\text {in }}$ 和 $\boldsymbol{U}_{\text {in }}$ 分别为 载荷和位移矢量。借助提取矢量 $\boldsymbol{L}$ (对应驱动方向的 单位矢量), 驱动位移可表述为 


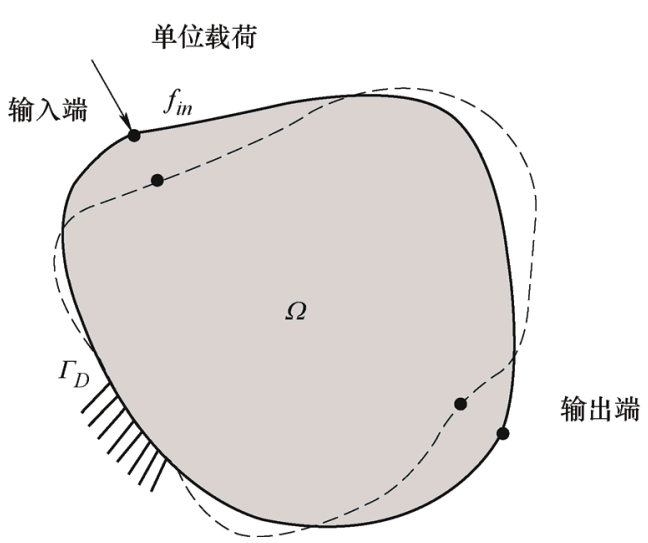

图 1 典型柔性机构示意图

$$
U_{\text {obj }}=\boldsymbol{L}^{\mathrm{T}} \boldsymbol{U}_{\text {in }}
$$

式中, $U_{\mathrm{obj}}$ 为驱动位移幅值, 记为标量形式。

以最大化驱动位移为设计目标的柔性机构设计 属于典型的非凸优化问题。后文考虑的典型位移反 向器设计中, 驱动位移随着机构拓扑构型的演化, 作用方向会发生变化, 难以直接应用采用离散拓扑 设计变量 $(0 / 1)$ 的双向渐进结构优化法开展相应的设 计。本文参照夏凉等 ${ }^{[38]}$ 的设计策略, 在式(2)中引入 一项动态衰减的机构综合柔顺度, 将目标函数改 写为

$$
\min J=-U_{\text {obj }}+\eta \cdot C_{\text {joint }}
$$

式中, $\eta=\delta^{\mathrm{i}}$ 为阻尼系数, $\delta$ 为 $0 \sim 1$ 之间的常数, 一般取 0.9 0.95, 本文取 0.9 , 上标 $\mathrm{i}$ 为优化设计的 迭代步数; $C_{\text {joint }}$ 为机构的综合柔顺度, 定义为如下 的输入和输出柔顺度之和

$$
C_{\text {joint }}=\boldsymbol{F}_{\text {in }}^{\mathrm{T}} \boldsymbol{U}_{\text {in }}+\boldsymbol{F}_{\text {out }}^{\mathrm{T}} \boldsymbol{U}_{\text {out }}
$$

式中, $\boldsymbol{F}_{\text {out }}$ 为输出端与驱动方向一致的单位虚拟激 励载荷, $\boldsymbol{U}_{\text {out }}$ 为该虚拟激励载荷作用下的机构的位 移响应。

由式(3)可见，通过逐步(设计迭代步)衰减综合 柔顺度的贡献, 目标函数由刚度设计逐渐演化至驱 动设计; 在优化迭代的后期, 阻尼系数接近于零, 优化设计的目标函数即为驱动位移。

由于仅考虑驱动位移最大化的柔性机构设计易 形成铰链, 本文进一步地借鉴朱本亮等 ${ }^{[35]}$ 的设计策 略, 将式(3)改进为如下的加权的形式

$$
\min J=-\omega \cdot U_{\mathrm{obj}}+(1-\omega+\eta) C_{\text {joint }}
$$

式中， $\omega$ 为权重因子, 取值区间为 $0 \sim 1$ 。通过调节 权重因子的取值, 可灵活调控所设计柔性机构的驱 动和刚度性能, 有效避免铰链的形成。

\section{2 优化模型及灵敏度分析}

应用双向渐进结构优化法, 采用离散拓扑设计 变量, 材料体分比约束下的柔性机构设计的优化模 型列式如下

$$
\begin{array}{cl}
\text { find } & \boldsymbol{x}=\left[x_{1}, x_{2}, \cdots, x_{n}\right] \\
\min & J=-\omega \cdot U_{\text {obj }}+(1-\omega+\eta) C_{\text {joint }} \\
\text { s.t. } & \boldsymbol{K} \boldsymbol{U}_{\text {in }}=\boldsymbol{F}_{\text {in }} \\
& \boldsymbol{K} \boldsymbol{U}_{\text {out }}=\boldsymbol{F}_{\text {out }} \\
& V(\mathbf{x})=\sum x_{e} v_{e}=V^{*} \\
& x_{e}=0 \text { 或 } 1, e=1,2, \cdots, n
\end{array}
$$

式中, $\boldsymbol{x}$ 是拓扑设计变量; $n$ 是设计域内有限单元 的总数; $V(\boldsymbol{x})$ 和 $V^{*}$ 分别是材料的实际和许用用量; $x_{e}$ 和 $v_{e}$ 分别是单元的设计变量和体积。

单元上定义的拓扑设计变量与单元的材料弹性 模量有以下线性关系

$$
E\left(x_{e}\right)=x_{e} E_{0}
$$

式中, $E_{0}$ 为实体材料的弹性模量。假设材料的泊松 比不变, 与拓扑设计变量不相关, 则单元的刚度矩 阵亦可表述为类似的线性关系

$$
\boldsymbol{k}\left(x_{e}\right)=x_{e} \boldsymbol{k}_{e}^{0}
$$

式中, $\boldsymbol{k}_{e}^{0}$ 为该单元充满实体材料时的刚度矩阵。

为实现结构拓扑构型的优化更新, 需要计算设 计变量相对于设计目标及约束的灵敏度信息，即一 阶偏导数。材料用量为设计变量的线性加和, 其对 应设计变量的偏导为

$$
\partial V / \partial x_{e}=v_{e}
$$

目标函数关于单元设计变量的偏导为

$$
\frac{\partial J}{\partial x_{e}}=-\omega \frac{\partial U_{\mathrm{obj}}}{\partial x_{e}}+(1-\omega+\eta) \frac{\partial C_{\mathrm{joint}}}{\partial x_{e}}
$$

利用伴随法 ${ }^{[6]}$, 可分别求得

$$
\begin{gathered}
\frac{\partial U_{\text {obj }}}{\partial x_{e}}=\boldsymbol{U}_{\text {out }}^{\mathrm{T}} \frac{\partial \boldsymbol{K}}{\partial x_{e}} \boldsymbol{U}_{\mathrm{in}}=\boldsymbol{u}_{\mathrm{out}, e}^{\mathrm{T}} \boldsymbol{k}_{e}^{0} \boldsymbol{u}_{\mathrm{in}, e} \\
\frac{\partial C_{\mathrm{joint}}}{\partial x_{e}}=-\boldsymbol{u}_{\mathrm{in}, e}^{\mathrm{T}} \boldsymbol{k}_{e}^{0} \boldsymbol{u}_{\mathrm{in}, e}-\boldsymbol{u}_{\mathrm{out}, e}^{\mathrm{T}} \boldsymbol{k}_{e}^{0} \boldsymbol{u}_{\mathrm{ou}, e}
\end{gathered}
$$

式中, $\boldsymbol{u}_{\mathrm{in}, e}$ 和 $\boldsymbol{u}_{\mathrm{out}, e}$ 分别为对应外部激励为 $\boldsymbol{F}_{\mathrm{in}}$ 和 $\boldsymbol{F}_{\mathrm{out}}$ 的单元位移矢量。 
根据以上优化模型列式和推导的灵敏度计算格 式, 可应用双向渐进结构优化法标准化的变量更新 机制开展柔性机构设计; 详细的更新步骤和说明可 参看文献[28], 在此不再赘述。

\section{3 数值算例}

本节将通过位移反向器和微夹持机构两个典型 算例来展示所提设计策略的适用性和局限性。基于 线弹性小变形模型假设, 以下采用无量纲化处理, 实体材料的弹性模量为 1 , 泊松比为 0.3 。式(3)中的 阻尼系数中的参数设为 0.9 。为避免数值计算时刚度 矩阵的奇异性, 空材料单元的弹性模量设为 $10^{-9}$ 。 输入点施加载荷的大小为 2 (利用对称性, 取半模计 算设计时载荷即为 1$)$ 。为增加设计的稳定性, 通常 在柔性机构的输入和输出端添加弹簧 ${ }^{[3]}$, 以模拟驱 动工件时的刚度。以下算例中, 输入与输出端弹簧 刚度的比值设置为满材料初始构型于输入和输出端 分别施加单位激励载荷下的柔度之比。

\section{1 位移反向器}

如图 2 所示, 方形设计域的左侧边界的上端和 下端分别固定。左侧边界的中间点为载荷输入端, 右侧边界中点为输出端(驱动端), 驱动方向水平向 左。由于驱动位移方向与输入载荷方向相反, 该 柔性机构称为位移反向器。设计域划分成 $120 \times 120$ 个四节点单位边长的正方形平面应力单元, 输入 端等效弹簧刚度值设为 0.1 。初始构型假设设计域 内充满实体材料, 许用材料用量为初始构型材料 用量的 $30 \%$, 灵敏度过滤半径设定为单元边长的 3 倍, 渐进优化法中的进化率设为 $2 \%$ (材料去除率) 设计步)。

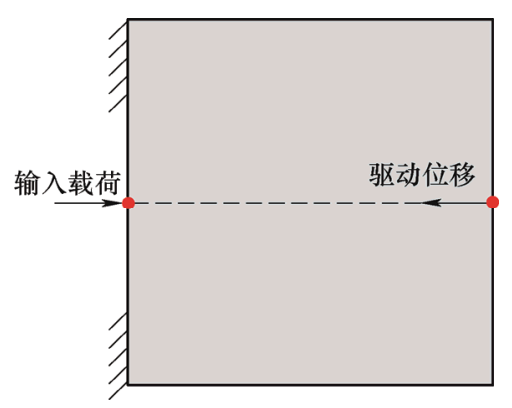

图 2 位移反向器设计问题

首先, 考虑当目标函数式(5)中权重因子为 1 时 $(\omega=1)$ 的情况, 即仅以驱动端位移作为优化目标开 展设计。如图 3 所示, 拓扑构型的演化进程平顺, 大约通过 30 个设计迭代步即可得到轮廓清晰、近似 于最终设计(图 3f)的中间设计拓扑构型(图 3d)。图 4
展示的驱动位移和目标函数的历史迭代曲线, 亦证 明整体拓扑构型演化进程的平顺性; 其中，在第 15 和 16 设计迭代步时, 机构的驱动方向发生改变, 拓 扑构型未显现明显震荡、构型演化过渡平稳。进一 步地, 通过比较应用变密度法 ${ }^{[3]}$ 设计的拓扑构型 (图 5a)与本文所提设计方法的设计构型(图 5b), 可 见两设计方案的拓扑构型十分接近, 且两者的驱动 性能亦几乎一致, 分别为: -1.03 和 -1.07 , 验证了 本文所提设计方法的有效性。

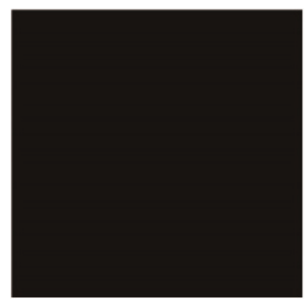

（a）初始结构

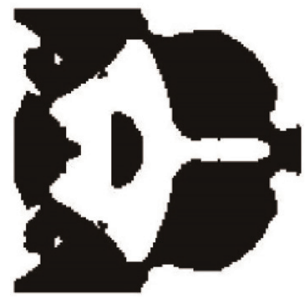

(c) 第20迭代步

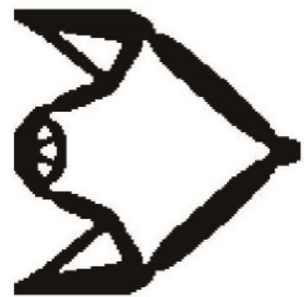

(e) 第 100 迭代步

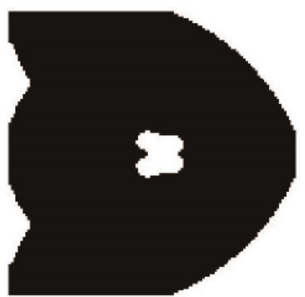

(b) 第10迭代步

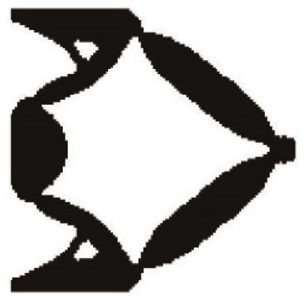

(d) 第30迭代步

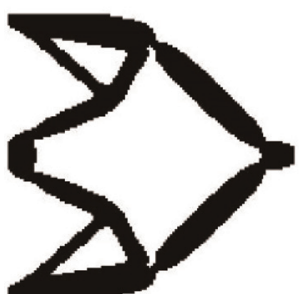

(f) 最终设计
图 3 反向器拓扑构型渐进式演化进程

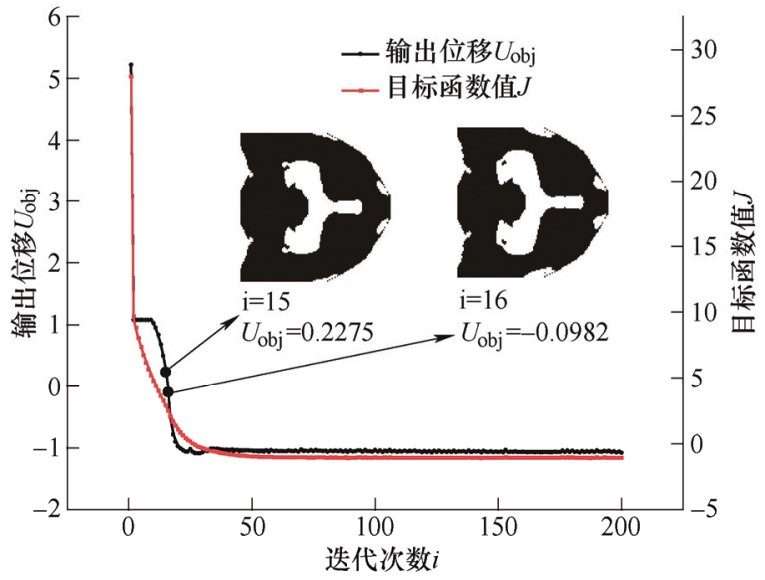

图 4 驱动位移和目标函数的历史迭代曲线 


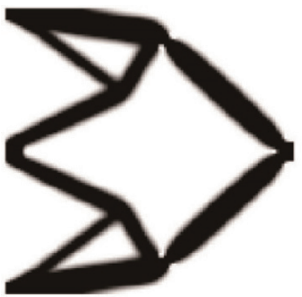

(a) 变密度法设计

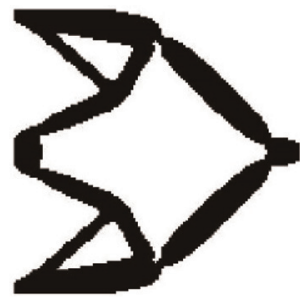

(a) 本图设计(图3f)

图 5 设计构型对比

接下来, 考虑当目标函数式(5)中权重因子取不 同值的情况, 即综合驱动位移和机构综合柔度作为 优化目标开展设计。图 6 展示了不同权重因子取值 对应设计得到的拓扑构型。由图可见, 随着权重因 子不断减小 (综合柔度占比逐渐增加), 设计的拓扑 构型逐渐趋于刚度设计, 图 6c、6d 驱动端位移水平 向右、无法实现预期的反向驱动。值得一提的是, 图 6a 对应的权重因子取 1 时设计构型中存在明显的 铰链结构形式(圆形标注区域)。通过适当的增加综 合柔度项的权重(权重因子取 0.8), 可实现如 图 $6 \mathrm{~b}$ 所示的避免了铰链、且兼顾刚度性能的机构设 计。图 7 为权重因子分别取 1 和 0.8 时的应力云图, 可见铰链的存在会导致显著的应力集中。图 8 为不 同权重因子取值对应设计的驱动位移和综合柔度, 由图可见通过调节权重因子可实现对机构的驱动和 刚度性能的灵活调控。实际应用过程中, 若机构的 驱动性能优先时, 权重因子在有效避免铰链现象的 前提下, 应可尽可能取大值; 相反, 若刚度性能优 先时, 则权重因子应尽量取小值。

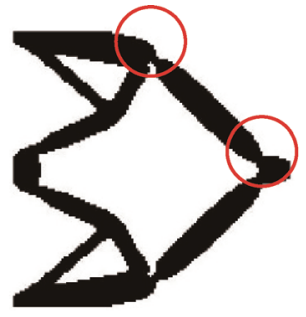

(a) 权重因子 $\omega=1$

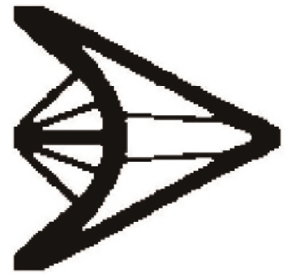

(c) 权重因子 $\omega=0.7$

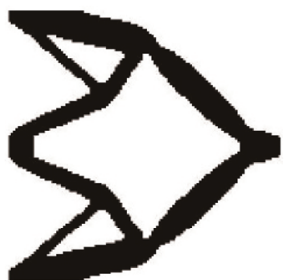

(b) 权重因子 $\omega=0.8$

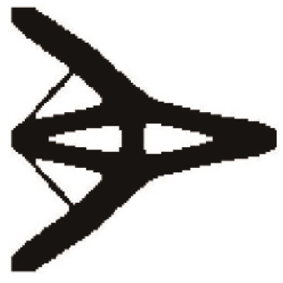

(d) 权重因子 $\omega=0.4$

图 6 不同权重因子取值对应的设计构型

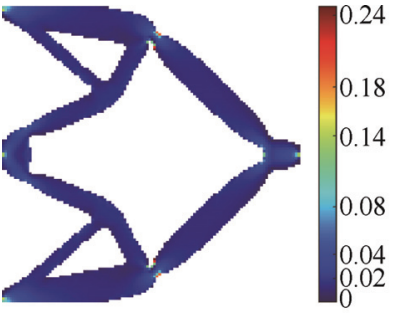

(a)权重因子 $\omega=1$

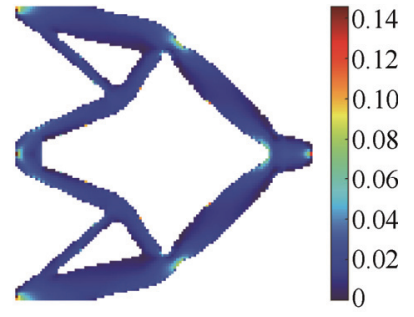

(b)权重因子 $\omega=0.8$
图 7 权重因子取值为 1 和 0.8 时设计构型的应力分布图

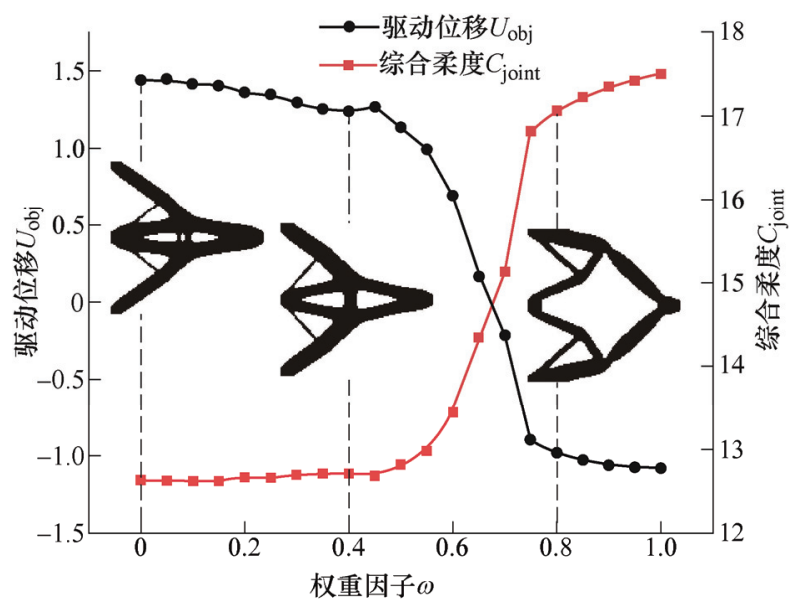

图 8 不同权重因子取值对应设计的驱动位移和综合柔度

最后, 为进一步地测试所提设计策略的稳健 性, 研究比对体分比设置和网格剖分精度对于设 计构型的影响。保持与图 3f 算例相同的网格尺寸 $(120 \times 120)$ 和权重因子 $(\omega=1)$, 材料用量体分比分 别为 $10 \%$ 和 $20 \%$ 的设计构型如图 $9 \mathrm{a} 、 9 \mathrm{~b}$ 所示，与 图 3f 构型较为一致且均实现反向驱动, 驱动位移 分别为 -0.51 和 -0.87 。图 9c、9d 分别为对应网格 剖分精度为 $60 \times 60$ 和 $240 \times 240$ 的设计构型, 与图 $3 \mathrm{f}$ 设计构型相比, 具有较好的一致性。此外, 本 算例的阻尼系数 $\delta$ 取值为 0.9 , 该系数主要功能在 于维系结构刚度设计向驱动设计的平稳演化。若 取值过大，则构型的演化趋向于刚度设计，难以 实现反向驱动的功能; 若取值过小, 则拓扑演化 进程震荡难以收敛。

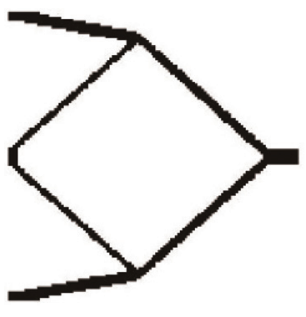

(a) 体分比 $10 \%$

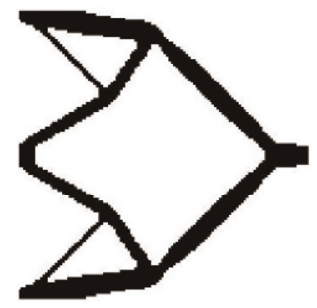

(b) 体分比 $20 \%$ 


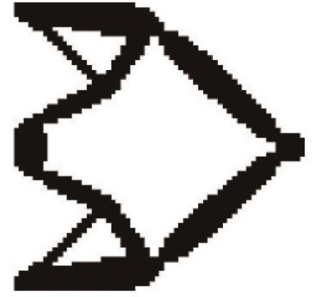

(c) 网络尺寸 $60 \times 60$

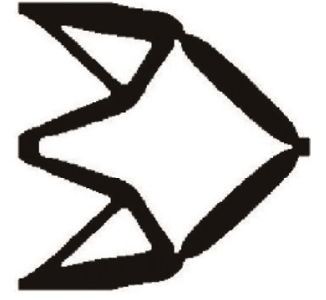

(d) 网络尺寸 $240 \times 240$
图 9 不同设计参数对应的拓扑设计方案

\section{2 微夹持器}

图 10 所示为微夹持器的设计域, 由边长为 160 的正方形平面于右侧删去边长为 $80 \times 40$ 的矩形区 域构成。与位移反向器类似, 微夹持器的左侧边 界的上端和下端分别固定。左侧边界中点处为驱 动载荷输入端, 驱动输出端位于设计域右侧红色 标识处的两个角点。由于该机构作夹持用, 两输 出点的驱动方向相对, 其中上角点驱动方向向下。 该设计域均匀离散成单位边长的四节点正方形平 面应力单元。许用材料用量为初始构型材料用量 的 $30 \%$, 过滤半径设置为 4 倍单元边长, 双向渐 进结构优化法中的进化率设为 $2 \%$, 输入端等效弹 簧刚度值为 0.1 。

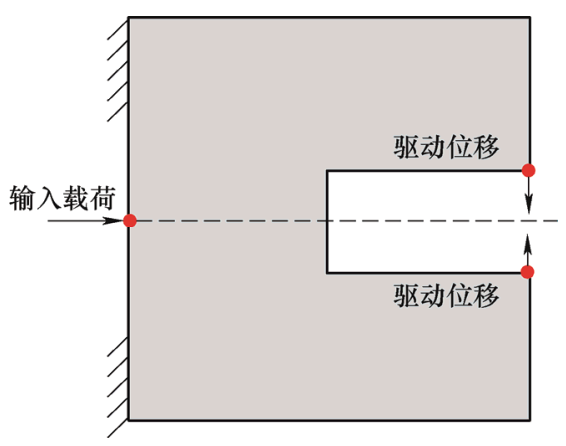

图 10 微夹持器设计问题

首先，考虑当权重因子为 1 时 $(\omega=1)$ 的情况， 即仅以驱动端位移为优化目标。微夹持器拓扑构 型的演化进程如图 11 所示, 在前期演化达到短暂 的稳定后在局部构型位置出现振荡现象, 难以收 玫。具体地, 在前期逐步减材过程中, 结构的拓 扑构型逐渐趋于稳定(图 10 中第 94 步); 之后, 自 第 150 步起拓扑构型发生振荡往复。图 10 中具体 展示了设计步 304、320、367 和 374 对应的拓扑 构型：第 304 步中构型中的细杆 $\mathrm{A}$ 与主体结构脱 离, 在后续设计步中逐渐消失; 相应的于杆 $\mathrm{B}$ 处 逐渐堆积材料, 后续设计步中又再一次分裂为类 似设计步 304 中两杆构型, 如此循环往复。大量
的设计测试表明, 当仅以驱动端位移最大化为设 计目标时，难以直接应用双向渐进结构优化法开 展微夹持器的构型设计。类似的收敛性问题亦见 于其他主流拓扑优化设计方法, 例如变密度法 ${ }^{[5]}$; 相对而言, 由于变密度法中变量的更新受限于设 定的步长, 其面向微夹持器设计的稳定性会较优 于双向渐进结构优化法。

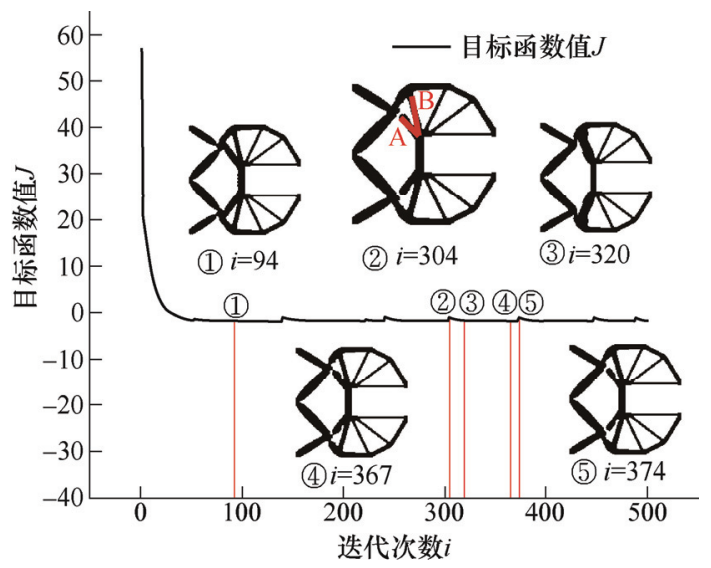

图 11 目标函数的历史迭代曲线

上述震荡难收敛的问题，可通过设置合适的 权重因子匹配驱动位移和机构综合柔度有效解 决。如图 $12 \mathrm{a}$ 所示, 当权重因子设为 0.7 时, 即可 应用双向渐进结构优化法稳定高效地设计得到具 有较优驱动性能且无明显铰链的机构拓扑构型。 当改变权重因子为 0.2 , 即进一步增大机构综合柔 度的占比, 设计得到图 $12 \mathrm{~b}$ 所示的趋于刚度设计 的构型, 无法实现夹持功能。为验证本文设计方 法的有效性，进一步地将本文的设计构型(图 12a) 与应用变密度法设计仅以驱动位移为优化目标的 优化构型(图 13a)作对比。由图可见，应用变密度 法设计纯驱动位移为优化目标的优化构型有明显 的铰链现象，相较之下，本文的设计构型结构不 存在铰链、材料分布更均匀。两者驱动位移分别 为 -1.67 和 -1.37 , 综合刚度方面分别为 40.30 和 37.22; 由此可见，本文设计构型的驱动性能虽略 有牺牲, 其刚度性能却得到增强, 改善了机构的 综合性能。

最后，为进一步地测试本文所提设计策略的稳 健性，保持与图 12 算例相同的网格尺寸 $(160 \times 160)$ 和权重因子 $(\omega=0.7)$, 研究比对体分比设置和网格 剖分精度对于设计构型的影响。图 14a 所示为材料 用量体分比为 $15 \%$ 的设计构型, 驱动位移为 -0.84 ; 图 14b 所示为网格剖分精度为 $80 \times 80$ 情况下的设计 构型, 与图 $13 \mathrm{~b}$ 的拓扑构型一致。 


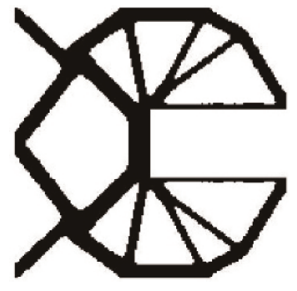

(a) 权重因子 $\omega=0.7$

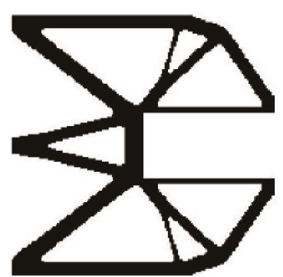

(b) 权重因子 $\omega=0.2$
图 12 不同权重因子取值对应的设计构型

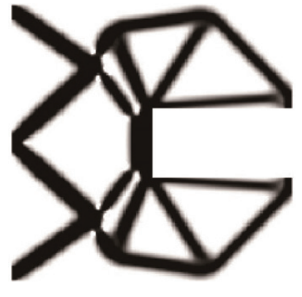

(a) 变密度法设计

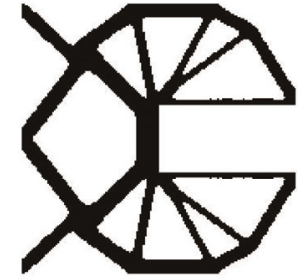

(b) 本文设计(图12a)
图 13 设计构型对比

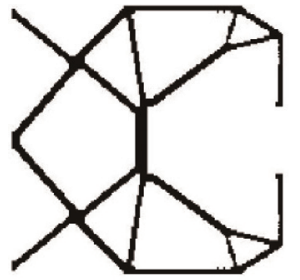

(a) 体积分数 $15 \%$

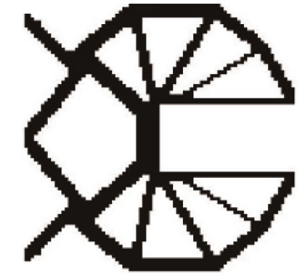

(b) 网格尺寸 $80 \times 80$
图 14 不同设计参数对应的拓扑设计方案

\section{4 结论}

本文提出一种综合驱动和刚度性能的设计目 标, 使得优化问题由刚度设计动态演化至驱动设 计, 进而实现了基于双向渐进结构优化法的柔性 机构设计。本文所提设计方法不仅保留了双向渐 进结构优化法概念简明、设计高效、构型清晰等 优点, 同时实现了对柔性机构的驱动和刚度性能 的灵活调控、有效抑制铰链的形成。所提设计目 标中的阻尼系数影响着动态演化的进程, 本文测 试算例显示该参数取值为 0.9 时设计构型最为合 理; 后续应用该演化策略设计开展其他柔性机构 问题设计时，可通过实验测试以选取合适的阻尼 系数。本文通过若干经典的二维算例验证了设计 方法的有效性, 后续函待将该设计方法拓展至三 维设计问题, 结合软体材料增材制造开展样件制 备与测试，同时进一步地开展考虑几何非线性的 驱动性能模拟。

\section{参 考 文 献}

[1] 于靖军, 郝广波, 陈贵敏, 等. 柔性机构及其应用研究 进展 [J]. 机械工程学报, 2015，51(13): 53-68.

YU Jingjun, HAO Guangbo, CHEN Guimin, et al. State-of-art of compliant mechanisms and their applications[J]. Journal of Mechanical Engineering, 2015, 51(13): $53-68$.

[2] 康荣杰, 杨铖浩, 杨名远, 等. 会思考的机器一一机械 智能 [J]. 机械工程学报, 2018，54(13): 15-24.

KANG Rongjie, YANG Chenghao, YANG Mingyuan, et al. Machines which can think-mechanical intelligence (MI)[J]. Journal of Mechanical Engineering, 2018, 54(13): $15-24$.

[3] HOWELL L. Compliant mechanisms[M]. New York: John Wiley \& Sons, 2001.

[4] KIM C J, KOTA S, MOON Y M. An instant center approach toward the conceptual design of compliant mechanisms[J]. Journal of Mechanical Design, 2006,

128(3): $542-550$.

[5] SAXENA A, ANANTHASURESH G K. Topology design of compliant mechanisms with strength consideration[J]. Mechanics of Structures and Mechanisms, 2003, 29(2): 199-221.

[6] SIGMUND O. On the design of compliant mechanisms using topology optimization[J]. Mechanics of Structures and Mechanisms, 1997, 25(4): 495-526.

[7] 于靖军, 裴旭, 毕树生, 等. 柔性铰链机构设计方法的 研究进展 [J]. 机械工程学报, 2010，46(13): 2-13.

YU Jingjun, PEI Xu, BI Shusheng, et al. State-of-arts of design method for flexure mechanisms[J]. Journal of Mechanical Engineering, 2010，46(13): 2-13.

[8] 占金青, 龙良明, 刘敏, 等. 基于最大应力约束的柔顺 机构拓扑优化设计 [J]. 机械工程学报, 2018, 54(23): 32-38.

ZHAN Jinqing, LONG Liangming, LIU Min, et al. Topological design of compliant mechanisms with maximum stress constraint[J]. Journal of Mechanical Engineering, 2018, 54(23): 32-38.

[9] BENDSØE M P. Optimal shape design as a material distribution problem[J]. Structural Optimization, 1989, 1: 193-202.

[10] SETHIAN J A, WIEGMANN A. Structural boundary design via level set and immersed interface methods[J]. Journal of Computational Physics, 2000, 163(2): 489-528.

[11] XIE Y M, STEVEN G P. A simple evolutionary procedure for structural optimization[J]. Computers \& Structures, 1993, 49(5): 885-896. 
[12] XIE Y M, STEVEN G P. Evolutionary structural optimization[M]. London: Springer, 1997.

[13] GUO X, ZHANG W, ZHONG W. Doing topology optimization explicitly and geometrically — a new moving morphable components Based Framework[J]. Journal of Applied Mechanics, 2014, 81(8): 081009.

[14] BENDSØE M P, SIGMUND O. Topology optimization: Theory, methods and applications[M]. Berlin: Springer, 2004.

[15] WANG M Y. Mechanical and geometric advantages in compliant mechanism optimization[J]. Frontiers of Mechanical Engineering in China, 2009, 4(3): 229-241.

[16] SIGMUND O. On the design of compliant mechanisms using topology optimization[J]. Mechanics of Structures and Machines, 1997, 25(4): 493-524.

[17] LAU G K, DU H, LIM M K. Use of functional specifications as objective functions in topological optimization of compliant mechanism[J]. Computer Methods in Applied Mechanics and Engineering, 2001, 190(34): 4421-4433.

[18] ZHU B, ZHANG X, ZHANG H, et al. Design of compliant mechanisms using continuum topology optimization: A review[J]. Mechanism and Machine Theory, 2020, 143: 103622.

[19] CHU S, GAO L, XIAO M, et al. Stress - based multi - material topology optimization of compliant mechanisms $[\mathrm{J}]$. International Journal for Numerical Methods in Engineering, 2018, 113: 1021-1044.

[20] LIU M, ZHAN J Q, ZHU B L, et al. Topology optimization of compliant mechanism considering actual output displacement using adaptive output spring stiffness[J]. Mechanism and Machine Theory, 2020, 146: 103728.

[21] 朱本亮. 基于水平集方法的分布式柔顺机构拓扑优化 设计研究[D]. 广州: 华南理工大学, 2014.

ZHU Benliang. Topology optimization of hinge-free compliant mechanisms using a level set method[D]. Guangzhou: South China University of Technology, 2014.

[22] RAHMATALLA S, SWAN C C. Sparse monolithic compliant mechanisms using continuum structural topology optimization[J]. International Journal for Numerical Methods in Engineering, 2005, 62(12): 1579-1605.

[23] SIGMUND O. Morphology-based black and white filters for topology optimization[J]. Structural and Multidisciplinary Optimization, 2007, 33(4): 401-424.

[24] POULSEN T A. A new scheme for imposing a minimum length scale in topology optimization[J]. International Journal for Numerical Methods in Engineering, 2003,
57(6): 741-760.

[25] WANG N, ZHANG X. Compliant mechanisms design based on pairs of curves[J]. Science China Technological Sciences, 2012, 55(8): 2099-2106.

[26] NISHIWAKI S, FRECKER M I, MIN S, et al. Topology optimization of compliant mechanisms using the homogenization method[J]. International Journal for Numerical Methods in Engineering, 1998, 42(3): 535-559.

[27] LAU G K, DU H, LIM M K. Convex analysis for topology optimization of compliant mechanisms[J]. Structural and Multidisciplinary Optimization, 2001, 22(4): 284-294.

[28] RAHMATALLA S, SWAN C C. Sparse monolithic compliant mechanisms using continuum structural topology optimization[J]. International Journal for Numerical Methods in Engineering, 2005, 62(12): 1579-1605.

[29] LI Y, HUANG X, XIE Y M, et al. Evolutionary topology optimization of hinge-free compliant mechanisms[J]. International Journal of Mechanical Sciences, 2014, 86: 69-75.

[30] HUANG X, XIE Y M. Bi-directional evolutionary topology optimization of continuum structures with one or multiple materials[J]. Computational Mechanics, 2009, 43(3): 393-401.

[31] XIA L, XIA Q, HUANG X, et al. Bi-directional evolutionary structural optimization on advanced structures and materials: a comprehensive review[J]. Archives of Computational Methods in Engineering, 2016, 25: 437-478.

[32] 于靖军, 周强, 毕树生, 等. 基于动力学性能的全柔性 机构优化设计[J]. 机械工程学报, 2003，39(8): 32-36. YU Jingjun, ZHOU Qiang, BI Shusheng, et al. Optimal design of a fully compliant mechanism based on its dynamic characteristics[J]. Journal of Mechanical Engineering, 2003, 39(8): 32-36.

[33] ZHU J H, ZHANG W H, XIA L. Topology optimization in aircraft and aerospace structures design[J]. Archives of Computational Methods in Engineering, 2015, 23(4): $1-28$.

[34] 杜义贤, 尹鹏, 李荣, 等. 兼具吸能和承载特性的梯度 结构宏细观跨尺度拓扑优化设计 [J]. 机械工程学报， 2020, 26(7): 171-180.

DU Yixian, YIN Peng, LI Rong, et al. Macro and micro trans-scale topological optimization design of gradient structure with both energy absorption and load-bearing characteristics $[\mathrm{J}]$. Journal of Mechanical Engineering, 2020, 26(7): 171-180.

[35] ZHU B, ZHANG X, ZHANG $\mathrm{H}$, et al. Design of 
compliant mechanisms using continuum topology optimization: A review[J]. Mechanism and Machine Theory, 2020, 143: 103622.

[36] ANSOLA R, VEGUERÍA E, CANALES J, et al. A simple evolutionary topology optimization procedure for compliant mechanism design[J]. Finite Elements in Analysis and Design, 2007, 44: 53-62.

[37] HUANG X, LI Y, ZHOU S W, et al. Topology optimization of compliant mechanisms with desired structural stiffness[J]. Engineering Structures, 2014, 79(15): 13-21.
[38] XIA L, BREITKOPF P. Design of materials using topology optimization and energy-based homogenization approach in Matlab[J]. Structural and Multidiplinary Optimization, 2015, 52: 1229-1241.

作者简介: 何健, 男, 1995 年出生。主要研究方向为柔性机构和压电结 构拓扑优化。

E-mail: m201870595@hust.edu.cn

夏凉(通信作者), 男, 1989 年出生, 博士, 副教授, 博士研究生导师。 主要研究方向为先进结构优化设计方法与应用。

E-mail: xialiang@hust.edu.cn 